\title{
A comprehensive checklist of the earthworms (Annelida: Clitellata: Megadrili) of Sri Lanka, a component of the Western Ghats - Sri Lanka biodiversity hotspot
}

\author{
Sasankan Prasanth Narayanan ${ }^{1}$, Shailja Kumari², Vijo Thomas Kurien ${ }^{3}$, \\ Ambattu Paili Thomas ${ }^{1}$, Rahul Paliwal ${ }^{4}$, Jatinder Mohan Julka ${ }^{2}$
}

1 Advanced Centre of Environmental Studies and Sustainable Development, Mahatma Gandhi University, Priyadarsini Hills, Kottayam - 686560, Kerala, India

2 School of Biological and Environmental Sciences, Faculty of Basic Sciences, Shoolini University, Solan - 173 212, Himachal Pradesh, India

3 Department of Zoology, CMS College, Kottayam - 686 001, Kerala, India

4 House No 77/62, Mansarovar, Jaipur - 302020, Rajasthan, India

Corresponding author: Sasankan Prasanth Narayanan (narayanankc@gmail.com)

Received 24 July 2020 | Accepted 11 December 2020 | Published 30 June 2021

Citation: Narayanan SP, Kumari S, Kurien VT, Thomas AP, Paliwal R, Julka JM (2021) A comprehensive checklist of the earthworms (Annelida: Clitellata: Megadrili) of Sri Lanka, a component of the Western Ghats - Sri Lanka biodiversity hotspot. Travaux du Muséum National d'Histoire Naturelle “Grigore Antipa” 64(1): 7-36. https://doi. org/10.3897/travaux.64.e56877

\begin{abstract}
A comprehensive checklist of the earthworm species known from Sri Lanka, an island country in the Indian subcontinent, is presented. In total, 81 species and subspecies belonging to 20 genera and 8 families are recorded. Of these, 58 are Sri Lanka endemics, 2 near endemics and 21 exotics. The checklist includes the type locality, any significant subsequent generic placements, and the distributional pattern with province and districts for each species/subspecies within Sri Lanka.
\end{abstract}

\section{Keywords}

endemic, Horton plains, Indian subcontinent, Megascolex, Notoscolex, taxonomy. 


\section{Introduction}

Sri Lanka is an island nation in the shallow Indian continental shelf, spread over an area of $65,584 \mathrm{~km}^{2}$ (between $9^{\circ} 50^{\prime}$ to $5^{\circ} 55^{\prime} \mathrm{N}$ and $81^{\circ} 53^{\prime}$ to $79^{\circ} 31^{\prime} \mathrm{E}$ ). This forms the second largest island in the Indian Ocean after Madagascar (Samaranayake and Moyle 1982; Illangasinghe et al. 1999; Szederjesi et al. 2019) (Fig. 1). This continental island is very rich in biodiversity with high endemism; most of the endemic flora and fauna are confined to the wet zone natural ecosystems of the country (Gunatilleke et al. 2008). The Western Ghats mountain range of the southwest portion of India, along with Sri Lanka, is represented as one of the important biodiversity hotspot in the world (Myers et al. 2000; Mittermeier et al. 2011). Present day Sri Lankan Island has been a part of the Indian plate during the Gondwanan break-up and drifted away in Miocene from the mainland India to form an island (Biswas 2008). The Sri Lanka Island had several extended periods of land connection with mainland India during the Burdigalian age ( 20-16 Mya) and the recent Pleistocene epoch as part of a series of glacial periods (Bossuyt et al. 2004; Epa et al. 2012; Sudasinghe et al. 2020).

Topographically, Sri Lanka can be divided into four regions namely, i) highland, situated in the south centre, ii) surrounding intermediate region of uplands, ridges and valleys at a lower elevation, iii) former region is in turn bounded by an outer or lower zone of low lands, and iv) surrounded by a coastal fringe consisting of sandbars, lagoons and islets (Illangasinghe et al. 1999). The north region and most of the eastern parts of the country is drier (Lindström 2011). Situated north of equator, the general climate of Sri Lanka can be described as tropical, with uniformly high temperatures throughout the year without major seasonal changes; the mean annual temperature in the lowland is around $27^{\circ} \mathrm{C}$ but at the central highlands it is $<15^{\circ} \mathrm{C}$ (Illangasinghe et al. 1999; Lindström, 2011). Sri Lanka receives the monsoon rainfall, the average annual rainfall is about $2000 \mathrm{~mm}$ but it is not uniform over geographical area of the island (Illangasinghe et al. 1999). The south-western part of the country receives most rainfall, especially between May and October (Lindström 2011). Based on the amount of rainfall the country has been divided into three different ecological zones viz., dry $(<1900 \mathrm{~mm}$ rainfall), intermediate $(1900-2540 \mathrm{~mm})$ and wet zones $(2540-5686 \mathrm{~mm})$ (Perera 2001). On account of Sri Lanka's topographic and climatic heterogeneity it has rich ecosystem diversity, among these most important with relation to the earthworms are the following - coastal ecosystems, natural/semi-natural grasslands, and various kinds of natural forests (Gunatilleke et al. 2008). Forests still cover 25\% of the Sri Lanka's total land area (Gunawardene et al. 2007) and most of it is situated in the dry zone (Perera 2001). Major forest types are mangroves, tropical forest groups such as lowland wet evergreen, moist evergreen, dry mixed evergreen, submontane and montane forests and thorn scrub forests (Gunatilleke et al. 2008). The tropical rain forests are found only in the southwest quarter of the country and much of these are fragmented (Gunawardene et al. 2007).

Taxonomic studies on the earthworms of Sri Lanka were started in the second quarter of the $19^{\text {th }}$ century by the description of Megascolex caeruleus by Robert 


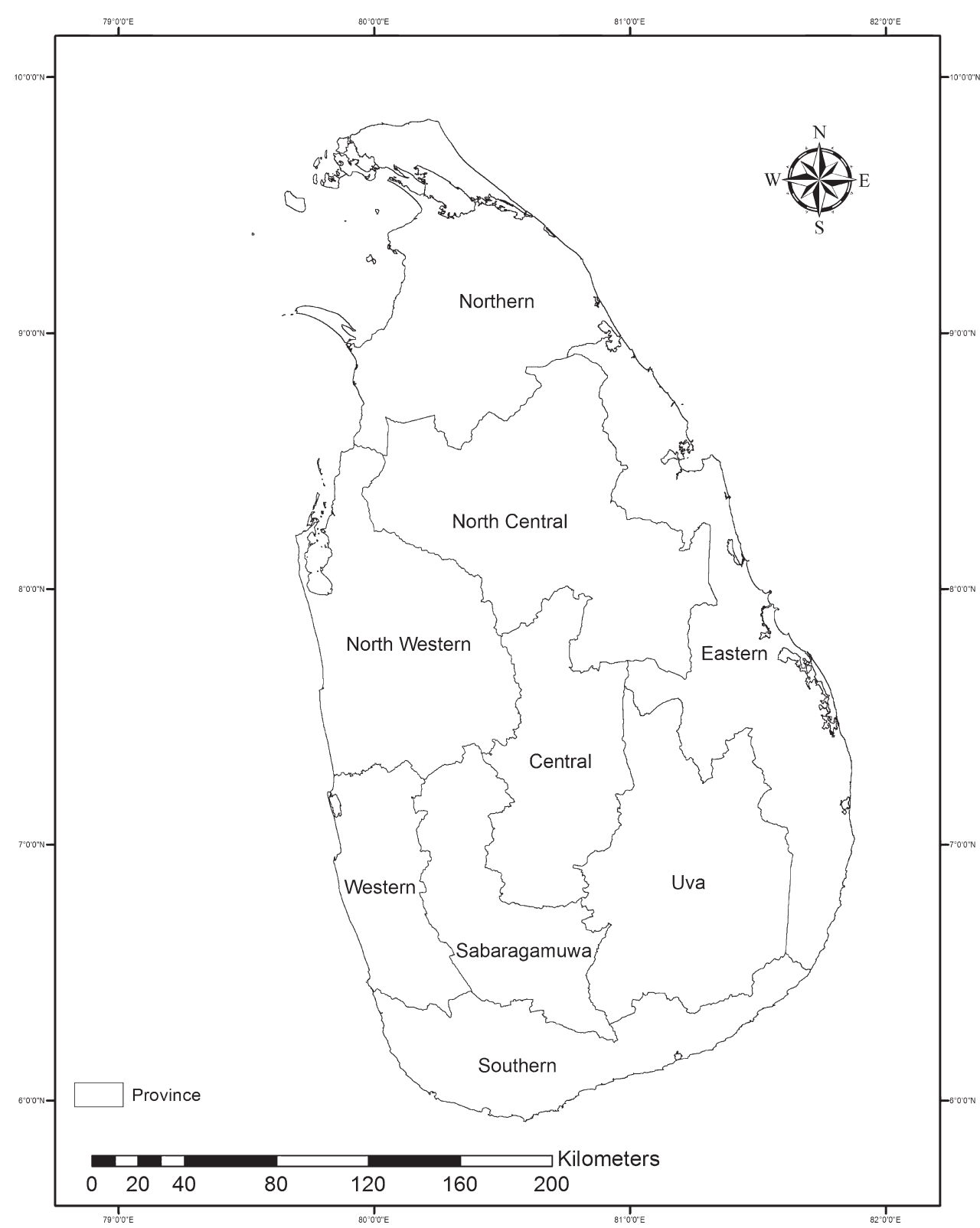

Figure 1. Sri Lanka: location and provinces.

Templeton in the year 1844. Eventually this represents the very first species scientifically described from the Indian subcontinent. Studies on the earthworm fauna in Sri Lanka had been very uneven since Templeton's discovery of the first earthworm. The initial active period, though brief, was towards the third quarter of the nineteenth century by Schmarda (1861). He described 4 new species from the island 
viz., Perichaeta brachycycla (now Megascolex brachycyclus), Perichaeta cingulata ( $M$. cingulatus), Perichaeta leucocycla (M. leucocyclus) and Perichaeta viridis (Megascolex ? viridis), of which, generic status of Perichaeta viridis is still uncertain. Towards the end of the nineteenth century (1876-1900), 26 new species were described from Sri Lanka (Beddard 1886, 1890; Rosa 1892, 1894; Michaelsen 1897, 1899), followed by another active period from 1901-1925, during which 23 new species were described (Michaelsen 1903, 1907, 1909, 1910; Stephenson 1913, 1915, 1916) (Fig. 2) and the sporadic period during 1926-1948 (Gates 1941, 1945a). Michaelsen is the pioneer among the taxonomists worked on the islands earthworm fauna, who described 30 new valid species, followed by Stephenson (13 species), Gates (8), Schmarda (4), Rosa and Beddard (3 each) and Templeton (1 species). In this moment it is worth to note that no nationwide taxonomical studies on earthworm were carried out after the independence of Sri Lanka from the colonial powers. Only three published materials are available on Sri Lankan earthworm fauna since its independence, they are of Samaranayake (2013), Wikramaratne and Krishnarajah (2013) and Szederjesi et al. (2019). First two were short-term studies on the diversity and ecology and not pure taxonomical, and the classification they used were of old and published in a symposium proceeding. Even though, Samaranayake (2013) added 4 exotic species, which were not reported earlier from the country.

Despite the high earthworm diversity, a complete list of earthworms of Sri Lanka has not been published after Stephenson's (1923) comprehensive work on the Oligochaeta of India and neighboring countries. Moreover, many changes in

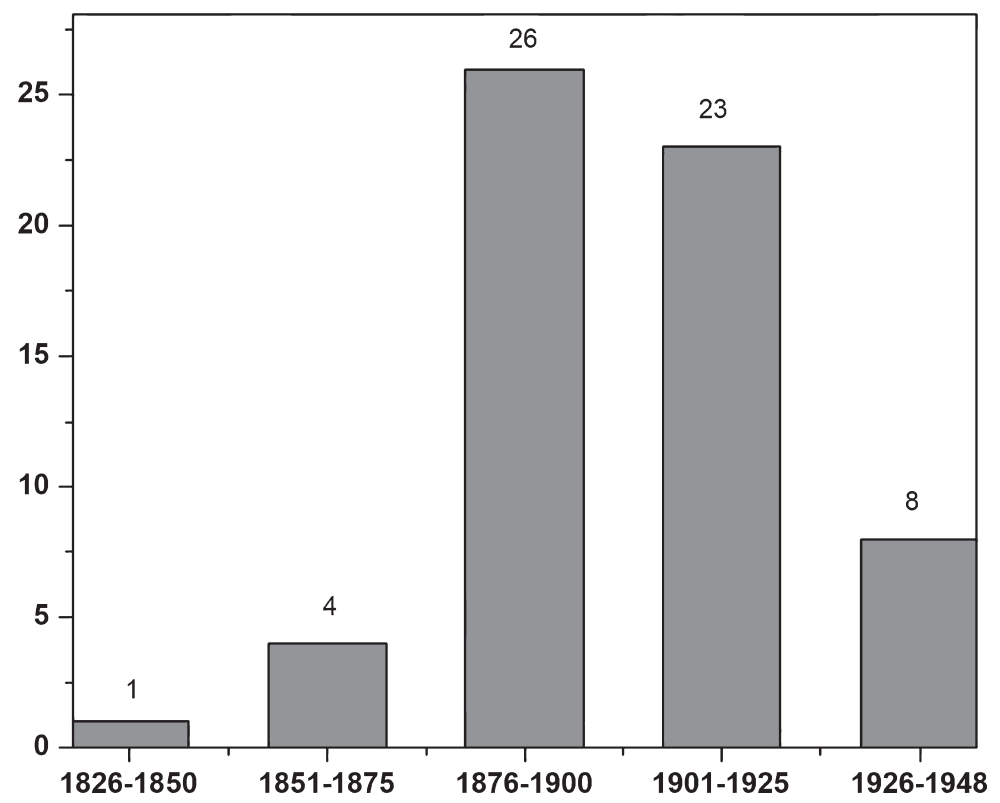

Figure 2. Valid earthworm species described from Sri Lanka at different periods. 
nomenclature and taxonomy of earthworms have occurred since Stephenson (1923). The aim of this study is, therefore, to prepare a checklist that updates nomenclature and distribution of Sri Lankan earthworms, and to provide a base for further investigations. Apart from their importance in identification, checklists form a source of useful information on the species distribution and endemicity, so that conservation strategies can be developed (Narayanan et al. 2016). As a result, 81 valid species and subspecies are recorded. Much is still to be learned about the earthworm diversity of the region. In this context, it is therefore hoped that this checklist shall be useful as most of Sri Lankan native earthworm species are associated with various habitats in the highlands and rain forests, which are among the most threatened ecosystems today.

\section{Methods}

This checklist is based on an exhaustive review of the entire literature on the earthworm fauna of Sri Lanka. The species entries include - 1) scientific name along with author and year of publication; 2) original and pertinent literature; 3 ) synonyms (mainly from the original literature and in the case of exotic species, from Blakemore (2012); 4) type locality (TL); 5) type material (TM) information on the type specimens of each species is from Reynolds and Wetzel (2017), unless reference is specified; 6) distribution (D) within Sri Lanka is arranged as province $(\mathbf{P})$ (ordered from north to south) followed by districts (Dt) (listed alphabetically) together with references (if a locality record is just a region, e.g. Sri Lanka, the locality is written as in the original publication); 7) distribution elsewhere (E), if applicable; and 8) remarks (R) on the taxonomic status of species, if necessary. Over the period of time, the names or spellings of collected localities have changed and this has created confusion about the distributional records of a number of species. In such cases the new name, as found in the literature, is given in brackets after the old name.

The museum abbreviations are as follows:

BMNH = British Museum of Natural History, London - United Kingdom

MHNG = Muséum d'histoire naturelle Genève, Geneva - Switzerland

MNHN = Museum National d'Histoire Naturelle Laboratoire de Zoologie (Vers), Paris - France

MNHU = Zoologisches Museum für Naturkunde der Humboldt Universität, Berlin - Germany

MZUT $=$ Museo ed Istituto di Zoologia Sistematica dell Università di Torino, Turin - Italy

NHRS = Naturhistoriska Riksmuseet Sektionen for Envertebratzoologi, Stockholm - Sweden

RNHL = Rijksmuseum van Natuurlijke Histoire, Leiden - The Netherlands

USNM = United States National Museum, Washington DC - USA

WNHM $[=\mathrm{NHMV}]=$ Naturhistorisches Museum Wien, Vienna - Austria 
ZMUH = Zoologisches Institut und Zoologisches Museum Universität, Hamburg - Germany

ZSIC = Zoological Survey of India, Calcutta (Kolkata) - India.

The classification of megascolecoid earthworms at the family level is not yet stabilized (Fender and McKey-Fender 1990; Blakemore 2005, 2013; Pavlíček et al. 2012; Narayanan et al. 2016). Blakemore's (2013) recent proposal to place megascolecoid earthworm taxa among several subfamilies appears to be a step in the right direction. Generally we have followed the classification as given by Gates (1959, 1972). However, based on Blakemore (2013), genera Argilophilus and Pontodrilus formerly of the family Acanthodrilidae have been placed in family Megascolecidae. Even though Dichogaster genus is placed under the family Benhamiidae (James and Gamiette 2016; Tiwari et al. 2020), however, previously it was in the Octochaetidae (Julka 1988). According to Gates (1960) Indo-Sri Lankan species of Notoscolex were not congeneric with those of Austro-New Zealand section of the genus. The genus Notoscolex Fletcher, 1886 belongs to the Australia-New Zealand region (Blakemore 2000). However, we have included Sri Lankan species of the genus under Notoscolex Fletcher, 1886 (sensu lato), following Stephenson (1923). Blakemore (2007a) placed Nellogaster from Sri Lanka in Notoscolex, but recently Szederjesi et al. (2019) retained it in Nellogaster; hence we followed the same. Species recorded only from Sri Lanka are considered endemic, whereas those with extended distribution to neighbouring India are referred to as near endemic. Exotic species are those, which have been introduced in Sri Lanka from other countries or zoogeographical regions.

\section{Checklist}

\section{Family MONILIGASTRIDAE}

\section{Drawida friderici (Michaelsen, 1897)}

Moniligaster friderici Michaelsen, 1897: 169.

Drawida friderici (Michaelsen): Michaelsen 1900: 115.

TL. Trincomali (Trincomalee), Sri Lanka; TM. ZMUH 4641; D: P. Eastern: Dt.

Trincomalee: Trincomali (Trincomalee) (Michaelsen 1897); R. endemic.

\section{Drawida pellucida bournei (Michaelsen, 1897)}

Moniligaster bournei Michaelsen, 1897: 167.

Moniligaster pauli Michaelsen, 1897: 171.

Drawida bournei (Michaelsen): Michaelsen 1900: 116.

Drawida pellucidus vr. bournei (Michaelsen): Michaelsen 1910: 50.

Drawida pellucida bournei (Michaelsen): Blakemore 2007a: 7.

TL. Probably Peradeniya, Sri Lanka; TM. Typus amissus, cotypus MNHU

7382; D. P. Eastern: Dt. Trincomalee: Kaniya (Kanniya) (Michaelsen 1910), 
Trincomali (Trincomalee) (Michaelsen 1900); P. Central: Dt. Kandy: Candy (Kandy) (Michaelsen 1897, 1900), Peradeniya (Michaelsen 1897); P. Western: Dt. Colombo: Colombo museum garden (Michaelsen 1899, 1900); P. Southern: Dt. Galle: Bentota (Michaelsen 1910), Vakvalla (Wakwella) (Michaelsen 1910); R. endemic.

\section{Family LUMBRICIDAE}

\section{Aporrectodea caliginosa (Savigny, 1826)}

Enterion caliginosum Savigny, 1826: 180.

Enterion carneum Savigny, 1826: 180.

Helodrilus (Allolobophora) caliginosus (part): Michaelsen 1900: 482.

Nicodrilus (Nicodrilus) caliginosus alternisetosus Bouché, 1972: 333.

Aporrectodea caliginosa caliginosa (part?) (Savigny): Easton 1983: 476.

For further synonyms see Blakemore (2012).

TL. Paris, France; TM. MHNG 3/77, MNHN; D. P. Western. Unspecified locality (Smaranayake 2013); R. exotic.

\section{Eisenia fetida (Savigny, 1826)}

Enterion fetidum Savigny, 1826: 182.

Lumbricus semifasciatus Burmeister, 1835: 3.

Allolobophora (Notogama) foetida (Savigny): Rosa 1893: 424.

Eisenia fetida (Savigny): Michaelsen 1903: 136.

Helodrilus (Eisenia) foetidus (Savigny): Michaelsen 1913: 551.

Allolobophora (Eisenia) fetida (Savigny): Stephenson 1923: 499.

Eisenia fasciata Backlund, 1948: 1.

Further synonymies in Blakemore (2012).

TL. Paris, France; TM. MNHN; D. P. Western. Unspecified locality (Smaranayake 2013); R.exotic.

\section{Octolasion cyaneum (Savigny, 1826)}

Enterion cyaneum (part) Savigny 1826: 181.

Allolobophora studiosa Michaelsen, 1890: 50.

Octolasion cyaneum (Savigny): Michaelsen 1900: 506.

Helodrilus (Dendrobaena) kempi Stephenson, 1922: 441.

For further synonyms see Blakemore (2012).

TL. Paris, France; TM. MNHN; D. P. Western. Unspecified locality (Smaranayake 2013); R. exotic. 


\section{Family ALMIDAE}

6. Glyphidrilus ceylonensis Gates, 1945

Glyphidrilus ceylonensis Gates, 1945a: 89.

TL. Pelmadulla, Sri Lanka; TM. Typus amissus; D. P. Sabaragamuwa: Dt.

Ratnapura: Pelmadulla (Gates 1945a); R. endemic.

\section{Family RHINODRILIDAE}

\section{Pontoscolex corethrurus (Müller, 1857)}

Lumbricus corethrurus Müller, 1857: 113.

Pontoscolex arenicola Schmarda, 1861: 11.

Urochaeta hystrix Perrier 1872: 142.

Pontoscolex hawaiiensis Beddard, 1895: 660.

Pontoscolexcorethrurus (Müller): Michaelsen 1903: 247.

Pontoscolex corethrurus (Müller): Samaranayake 2013: 38. (misspelt corathrurus).

Further synonymies in Blakemore (2012).

TL. Grass lawn behind the Museu de Ecologia Fritz Müller (former house of Fritz Müller), Blumenau, Santa Catarina, Brazil (Neotype) (James et al. 2019); Type. MZUSP 3532 (Neotype) (James et al. 2019); D. P. Central: Dt. Kandy: Kandy (Michaelsen 1909), Peradeniya (Michaelsen 1897, 1903; Gates 1945a), Dt. Matale: Suduganga (Stephenson 1926); P. Uva: Dt. Badulla: Avissavela (Michaelsen 1910); P. Sabaragamuwa: Dt. Ratnapura: Adams Peak (Michaelsen 1897), Palmadulla (Pelmadulla) (Gates 1945a), P. Western: Dt. Colombo: Colombo museum garden (Michaelsen, 1899), Dehiwala-Mount Lavinia (Szederjesi et al. 2019), Tannepita (Pannipitiya?) (Gates 1945a), Dt. Gampaha: Heneratgoda (Henarathgoda) (Gates 1945a), Dt. Kalutara: Matugama (Szederjesi et al. 2019), Moratuwa (Szederjesi et al. 2019), near the shore of Bolgoda Lake Wadduwa (Szederjesi et al. 2019), Dt. Unspecified (Samaranayake 2013); R. exotic. According to Stephenson (1915) juvenile Pontoscolex specimens from the West Haputale Estate (Uva province, Badulla district) would be of this species.

\section{Family EUDRILIDAE}

\section{Eudrilus eugeniae (Kinberg, 1867)}

Lumbricus eugeniae Kinberg, 1867: 98.

Eudrilus decipiens Perrier, 1871: 1176.

Eudrilus eugeniae (Kinberg): Michaelsen 1897: 246.

Further synonymies in Gates (1972), Blakemore (2010).

TL. St. Helena Island, South Atlantic; TM. NHRS 1237, Typus quoque locates in BMNH 1904.10.5.550; D. P. Central: Dt. Kandy: Kandy (Michaelsen 1897), Peradeniya (Michaelsen 1897, 1903); P. Western: Dt. Colombo: Colombo 
museum garden (Michaelsen 1899), Dt. Kalutara: Panadhure (Panadura) (Michaelsen 1910), Dt. Unspecified (Samaranayake 2013); P. Southern: Dt. Galle: Bentota (Michaelsen 1910); R. exotic.

\section{Family OCNERODRILIDAE}

\section{Nematogenia panamaensis (Eisen, 1900)}

Ocnerodrilus (Nematogenia) lacuum var. panamaensis Eisen, 1900: 127.

Nematogenia panamaensis (Eisen): Michaelsen 1900: 376.

For further synonyms see Gates (1972), Blakemore (2012).

TL. Panama, Panama; TM. Unknown (Nguyen et al. 2016); D. P. Central: Dt. Kandy: Peradeniya (Michaelsen 1903); R. exotic.

\section{Ocnerodrilus occidentalis Eisen, 1878}

Ocnerodrilus occidentalis Eisen, 1878: 10.

Ocnerodrilus tenellulus Gates, 1945b: 223.

Further synonyms in Blakemore (2012).

TL. Fresno County, USA; TM. MNHU 2363 Typus amissus, BMNH 1904: 10:5:231-6, but as per Nguyen et al. (2016) at USNM; D. P. Western: Dt. Kalutara: Panadhure (Panadura) (Michaelsen 1910); R. exotic.

\section{Family BENHAMIIDAE}

\section{Dichogaster affinis (Michaelsen, 1890)}

Benhamia affinis Michaelsen, 1890: 9.

Benhamia mexicana Rosa, 1891: 394.

Dichogaster affinis (Michaelsen): Michaelsen 1903: 16.

For furthur synonyms see Blakemore (2012).

TL. Quilimane, Mozambique; TM. ZMUH 303; D. P. North Central: Dt. Anuradhapura: Anuradhapura (Stephenson 1913); P. Central: Dt. Kandy: Peradeniya (Michaelsen 1903); R. exotic.

\section{Dichogaster annae (Horst, 1893)}

Benhamia annae Horst, 1893: 32.

Dichogaster annae (Horst): Michaelsen 1900: 347.

Dichogaster curgensis Michaelsen, 1922: 54.

Dichogaster (Diplothecodrilus) annae (Horst): Csuzdi 1995: 112.

Further synonyms see Blakemore (2012).

TL. Java, Indonesia; TM. RNHL 1798; D. P. Central: Dt. Kandy: Peradeniya (Michaelsen 1903); R. exotic.

\section{Dichogaster bolaui (Michaelsen, 1891)}

Benhamia bolavi (corr. bolaui) Michaelsen, 1891: 9. 
Dichogaster bolaui (Michaelsen): Michaelsen 1903: 16.

Dichogaster (Diplothecodrilus) bolaui (Michaelsen): Csuzdi 1995: 102.

Further synonyms in Blakemore (2012).

TL. Bergedorf $\left(53.48^{\circ} \mathrm{N}, 10.21^{\circ} \mathrm{E}\right)$, Germany; TM. ZMUH 285, BMNH 1924:3:1:244, MNHU 7334, MZUT 52, NHRS 1247, RNHL, USNM 34166; but according to Blakemore (2012), type was thought to be in the ZMUH but it is at BMNH: 1924:3.1.244; D. P. Central: Dt. Kandy: Peradeniya (Michaelsen 1903);

P. Western. Unspecified locality (Samaranayake 2013); R. exotic.

\section{Dichogaster saliens (Beddard, 1893)}

Microdrilus saliens Beddard, 1893: 683.

Dichogaster crawi Esien, 1900: 228

Dichogaster saliens (Beddard): Michaelsen 1903: 13.

Dichogaster (Diplothecodrilus) saliens (Beddard): Csuzdi 1996: 358.

TL. Not designated but specimens were found by Mr Thistleton Dyer at Kew Gardens in soil from Singapore, Java and Penang (Blakemore 2012); TM. BMNH 1904: 10:5:536-40; D. P. Central: Dt. Kandy: Peradeniya (Michaelsen 1903); R. exotic.

\section{Family MEGASCOLECIDAE}

\section{Amynthas corticis (Kinberg, 1867)}

Perichaeta corticis Kinberg, 1867: 102.

Perichaeta indica ceylonica Michaelsen, 1897: 246.

Pheretima heterochaeta (Michaelsen): Michaelsen 1910: 83.

Amynthas corticus (Sic lapsus pro corticis) (Kinberg): Sims and Easton 1972: 235.

Amynthas corticis (Kinberg): Easton 1982: 726.

Amynthas indica ceylonicus (Michaelsen): Blakemore 2007a: 23.

For full list of synonyms see Blakemore (2012).

TL. Oahu, Hawaii Island; TM. NHRS 1947; possibly immature (Blakemore 2012); D. P. Central: Dt. Nuwara Eliya: Adams Peak (Michaelsen 1897), Horton Plains (Stephenson 1915), Pattipola (Stephenson 1915); P. Sabaragamuwa: Dt. Ratnapura: Adams Peak (Michaelsen 1897), Bulutota at Adams Peak (Michaelsen 1910); P. Western: Dt. Kalutara: Panadhure (Panadura) (Michaelsen 1910); R. exotic.

\section{Amynthas gracilis (Kinberg, 1867)}

Nitocris gracilis Kinberg, 1867: 102.

Pheretima hawayana (Kinberg): Stephenson 1913: 271.

Perichaeta mandhorensis Michaelsen, 1892: 241. 
Amynthas gracilis (Kinberg): Sims and Easton 1972: 235.

For further synonyms see Blakemore (2012).

TL. Rio de Janeiro, Brazil; TM. NHRS 1944, but according to Nguyen et al. (2016) it is in RNHL; D. P. Central: Dt. Nuwara Eliya: Pattipola (Stephenson 1913); P. Western. Unspecified locality (Samaranayake 2013); R. exotic.

17. Argilophilus halyi (Michaelsen, 1899)

Megascolides halyi Michaelsen, 1899: 142.

Plutellus halyi (Michaelsen): Michaelsen 1900: 165.

Argilophilus halyi (Michaelsen): Blakemore 2007a: 28.

TL. Colombo museum garden, Sri Lanka; TM. Typus amissus; D. P. Western: Dt. Colombo: Colombo museum garden (Michaelsen 1899); R. endemic.

\section{Argilophilus singhalensis (Michaelsen, 1897)}

Megascolides singhalensis Michaelsen, 1897: 174.

Plutellus singhalensis (Michaelsen): Michaelsen 1900: 165.

Argilophilus singhalensis (Michaelsen): Blakemore 2007a: 28.

TL. Nuwara Eliya, Sri Lanka; TM. Typus amissus; D. P. Central: Dt. Nuwara Eliya: Nuwara Eliya (Michaelsen 1897); R. endemic.

\section{Lampito mauritii Kinberg, 1867}

Lampito mauritii Kinberg, 1867: 103.

Perichaeta luzonica Perrier, 1875:1044.

Megascolex armatus (Beddard): Michaelsen 1897: 210.

Megascolex mauritii (Kinberg): Michaelsen 1900: 227.

Lampito mauritii zeylanica Stephenson, 1913: 262.

Megascolex mauritii zeylanicus (Stephenson): Stephenson 1923: 260.

Lampito mauritii zeylanicus (Stephenson): Blakemore 2007a: 32. (misspelt zelandicus)

For full list of synonyms see Gates $(1938,1972)$ and Blakemore (2012).

TL. Mauritius; TM. NHRS 162; D. P. Northern: Dt. Jaffna: Belligamme (Valikamam, Valligamam, Valikaamam) (Michaelsen 1897), Jaffna (Michaelsen 1897; Gates 1945a); P. Eastern: Dt. Trincomalee: Kanthalai (Kantale, Kantalai) (Michaelsen 1910), Trincomali (Trincomalee) (Michaelsen 1897); P. North Central: Dt. Anuradhapura: Anuradhapura (Stephenson 1913); P. Central: Dt. Kandy: Peradeniya (Michaelsen 1910); Dt. Matale: Dambulla (Michaelsen 1897); P. Western: Dt. Colombo: Colombo museum garden (Michaelsen 1899), Dehiwala-Mount Lavinia (Szederjesi et al. 2019), Dt. Kalutara: Panadhure (Panadura) (Michaelsen 1910), unspecified locality (Wickramaratne and Krishnarajah 2013), Dt. Unspecified district and locality (Samaranayake 2013); P. Southern: Dt. Galle: Bentota (Michaelsen 1910), Vakvalla (Wakwella) (Michaelsen 1910); R. exotic. 
20. Megascolex acanthodriloides Michaelsen, 1897

Megascolex acanthodriloides Michaelsen, 1897: 235.

TL. Peradeniya, Sri Lanka; TM. Typus amissus; D. P. Central: Dt. Kandy: Peradeniya (Michaelsen 1897); P. Western: Dt. Gampaha: Heneratgoda (Henarathgoda) (Gates 1941); R. endemic.

21. Megascolex adami Michaelsen, 1910

Megascolex adami Michaelsen, 1910: 64.

TL. Bulutota $\left(6.433^{\circ} \mathrm{N}, 80.65^{\circ} \mathrm{E}\right)$, Sri Lanka; TM. Typus amissus; D. P. Sabaragamuwa: Dt. Ratnapura: Bulutota - Adams Peak (Michaelsen 1910); R. endemic.

22. Megascolex bifoveatus Stephenson, 1913

Megascolex bifoveatus Stephenson, 1913: 266.

TL. Pattipola, Sri Lanka; TM. ZSIC 5084; D. P. Central: Dt. Nuwara Eliya: Horton Plains (Stephenson 1915), Pattipola (Stephenson 1913); R. endemic.

23. Megascolex brachycyclus (Schmarda, 1861)

Perichaeta brachycycla Schmarda, 1861: 14.

Megascolex brachycyclus (Schmarda): Beddard 1895: 382.

TL. Ratnapura, Sri Lanka; TM. ?; D. P. Sabaragamuwa: Dt. Ratnapura: Ratnapura at the foot of Adam's peak (Schmarda 1861; Michaelsen 1900); R. endemic.

\section{Megascolex caeruleus Templeton, 1844}

Megascolex caeruleus Templeton, 1844: 89.

Pleurochaeta moseleyi (Templeton): Beddard 1882: 481.

TL. Alpine region of Ceylon (Sri Lanka); TM. Typus amissus; D. Alpine region of Ceylon (Sri Lanka) (Templeton 1844); P. Central: Dt. Kandy: Kandy (Beddard 1882; Bourne 1891; Michaelsen 1897), Peradeniya (Bourne 1891; Michaelsen 1897), Dt. Nuwara Eliya: Nuwara Eliya (Michaelsen 1897); R. endemic.

\section{Megascolex campester Stephenson, 1915}

Megascolex campester Stephenson, 1915: 78.

TL. Horton Plains, Sri Lanka; TM. ZSIC 6186; D. P. Central: Dt. Nuwara Eliya: Horton Plains (Stephenson 1915); R. endemic.

26. Megascolex ceylonicus (Beddard, 1886)

Perichaeta ceylonica Beddard, 1886: 89.

Megascolex ceylonica (Beddard): Beddard 1895: 385.

Megascolex ceylonicus (Beddard): Michaelsen 1900: 228.

TL. Unknown (Gates 1945a), Sri Lanka; TM. BMNH 1904.10.5.17, 1904.10.20.677-

81, Typus amissus BMNH1904.10.5.17, 1904.10.20.677-81; D. Ceylon (Sri Lanka) 
(Beddard 1886); P. Sabaragamuwa: Dt. Ratnapura: Ratnapura (Gates 1945a); R. endemic.

27. Megascolex cingulatus (Schmarda, 1861)

Perichaeta cingulata Schmarda, 1861: 14

Megascolex cingulatus (Schmarda): Beddard 1895: 382.

TL. East of Badulla, Sri Lanka; TM. MNH; D. P. Central: Dt. Kandy: Lady Blake's Drive - Kandy (Stephenson 1916), probably Peradeniya (Michaelsen 1900); P. Uva: Dt. Badulla: East of Badulla (Schmarda 1861), Avissavela (Michaelsen 1910); R. endemic.

28. Megascolex escherichi escherichi Michaelsen, 1910

Megascolex escherichi Michaelsen, 1910: 66.

Megascolex escherichi (Michaelsen): Stephenson 1923: 241.

Megascolex escherichi escherichi (Michaelsen): Blakemore 2007a: 33.

TL. Hidana near Peradeniya, Sri Lanka; TM. Typus amissus; D. P. Central: Dt. Kandy: Hidana near Peradeniya (Michaelsen 1910); R. endemic.

29. Megascolex escherichi papillifer Stephenson, 1915

Megascolex escherichi papillifer Stephenson, 1915: 77.

TL. Horton Plains, Sri Lanka; TM. ZSIC 6187, 6926; D. P. Central: Dt. Nuwara Eliya: Horton Plains (Stephenson 1915); R. endemic.

30. Megascolex funis Michaelsen, 1897

Megascolex funis Michaelsen, 1897: 210.

TL. Kandy (Michaelsen 1909), Sri Lanka; TM. Typus amissus; D. P. Central: Dt. Kandy: Kandy (Michaelsen 1909), probably in Peradeniya (Michaelsen 1897, 1900); R. endemic.

\section{Megascolex hakgallanus Gates, 1941}

Megascolex hakgallanus Gates, 1945a: 49.

TL. Hakgalla (Hakgala), Sri Lanka; TM. ?; D. P. Central: Dt. Nuwara Eliya: Hakgalla (Hakgala) (Gates 1941); R. endemic.

\section{Megascolex hortonensis Stephenson, 1915}

Megascolex hortonensis Stephenson, 1915: 83

TL. Horton Plains, Sri Lanka; TM. ZSIC 6928; D. P. Central: Dt. Nuwara Eliya: Horton Plains (Stephenson 1915); R. endemic. Closely related to M. kempi and M. quintus (Stephenson 1923). 
33. Megascolex insignis Michaelsen, 1910

Megascolex insignis Michaelsen, 1910: 78.

TL. Trivandrum (Thiruvananthapuram), India. TM. ZSIC 4176; D. P. Western:

Dt. Kalutara: Matugama (Szederjesi et al. 2019), near the shore of Bolgoda Lake Moratuwa (Szederjesi et al. 2019), Panadhure (Panadura) (Michaelsen 1910), Wadduma (Szederjesi et al. 2019); E. India (Michaelsen 1910); R. near endemic.

\section{Megascolex kempi Stephenson, 1915}

Megascolex kempi Stephenson, 1915: 84.

TL. Horton Plains, Sri Lanka; TM. Typus amissus; D. P. Central: Dt. Nuwara Eliya: Horton Plains (Stephenson 1915); R. endemic. Very closely related to $M$. quintus (Stephenson 1923).

\section{Megascolex leucocyclus (Schmarda, 1861)}

Perichaeta leucocycla Schmarda, 1861: 13

Megascolex leucocyclus (Schmarda): Michaelsen 1897: 215.

TL. Kandy, Sri Lanka; TM. ?; D. P. Central: Dt. Kandy: Kandy (Schmarda 1861; Michaelsen 1897), Dt. Nuwara Eliya: Newera-Ellia (Nuwara Eliya) (Schmarda 1861; Michaelsen 1897); R. endemic.

36. Megascolex longiseta Michaelsen, 1907

Megascolex longiseta Michaelsen, 1907: 163

TL. Nuwara Eliya, Sri Lanka; TM. Typus amissus; D. P. Central: Dt. Nuwara Eliya: Nuwara Eliya (Michaelsen 1907); R. endemic.

\section{Megascolex lorenzi Rosa, 1894}

Megascolex lorenzi Rosa, 1894: 5.

TL. Kandy, Sri Lanka; TM. ?; D. P. Central: Dt. Kandy: Kandy (Rosa 1894; Michalsen 1897), Peradeniya (Michaelsen 1910); R. endemic.

38. Megascolex multispinus Michaelsen, 1897

Megascolex multispinus Michaelsen, 1897: 221.

TL. Peradeniya?, Sri Lanka; TM. MNHU 7473 but Typus amissus; D. P. Central: Dt. Kandy: probably Peradeniya (Michaelsen 1897); R. endemic.

39. Megascolex nureliyensis Michaelsen, 1897

Megascolex nureliyensis Michaelsen, 1897: 232.

TL. Nuwara Eliya, Sri Lanka; TM. Typus amissus; D. P. Central: Dt. Nuwara Eliya: Nuwara Eliya (Michaelsen 1897), Horton Plains (Stephenson 1915); R. endemic. 
40. Megascolex pattipolensis Stephenson, 1913

Megascolex pattipolensis Stephenson, 1913: 265.

TL. Pattipola, Sri Lanka; TM. ZSIC 4894; D. P. Central: Dt. Nuwara Eliya: Pattipola (Stephenson 1913); R. endemic.

41. Megascolex peranellus Gates, 1945

Megascolex peranellus Gates, 1945a: 78.

TL. Hakgalla (Hakgala), Sri Lanka; TM. Typus perditus; D. P. Central: Dt. Nuwara Eliya: Hakgalla (Hakgala) (Gates 1945a); R. endemic.

42. Megascolex peranus Gates, 1941

Megascolex peranus Gates, 1945a: 52.

TL. Hakgalla (Hakgala) $\left(6.9266^{\circ} \mathrm{N}, 80.8215^{\circ} \mathrm{E}\right)$, Sri Lanka; TM. ?; D. P. Central:

Dt. Kandy: Peradeniya (Gates 1941); R. endemic. Species inquirenda.

\section{Megascolex pharetratus Rosa, 1894}

Megascolex pharetratus Rosa, 1894: 3.

TL. Kandy, Sri Lanka; TM. ?; D. P. Central: Dt. Kandy: Kandy (Rosa 1894; Michaelsen 1897); R. endemic.

\section{Megascolex quintus Stephenson, 1913}

Megascolex quintus Stephenson, 1913: 268.

TL. Pattipola, Sri Lanka; TM. ZSIC 5081; D. P. Central: Dt. Nuwara Eliya: Pattipola (Stephenson 1913); R. endemic.

\section{Megascolex sarasinorum Michaelsen, 1897}

Megascolex sarasinorum Michaelsen, 1897: 224.

TL. Trincomali (Trincomalee), Sri Lanka, but as per Gates (1945) type locality is undesignated; TM. BMNH 1904:10:5:475 but Typus amissus; D. P. Eastern: Dt. Trincomalee: Kaniya (Kanniya) (Michaelsen 1910), Mahavali Ganga (Mahaweli Ganga) (Michaelsen 1910), Trincomali (Trincomalee) (Michaelsen 1897); P. Central: Dt. Matale: plains north of Dambulla (Michaelsen 1897); P. Western: Dt. Colombo: Colombo (Gates 1945a); R. endemic.

46. Megascolex schmardae Michaelsen, 1897

Megascolex schmardae Michaelsen, 1897: 208.

TL. Ratnapura, Sri Lanka; TM. ?; D. P. Sabaragamuwa: Dt. Ratnapura: Ratnapura at the foot of Adam's peak (Michaelsen 1897); R. endemic.

47. Megascolex sextus Stephenson, 1913

Megascolex sextus Stephenson, 1913: 270.

TL. Pattipola, Sri Lanka; TM. ZSIC 5083; D. P. Central: Dt. Nuwara Eliya: Pattipola (Stephenson 1913, 1915); R. endemic. 
48. Megascolex singhalensis Michaelsen, 1897

Megascolex singhalensis Michaelsen, 1897: 227.

TL. Nuwara Eliya, Sri Lanka; TM. WNHM 3971; D. P. Central: Dt. Nuwara

Eliya: Nuwara Eliya (Michaelsen 1897); R. endemic.

49. Megascolex spectabilis Michaelsen, 1910

Megascolex spectablilis Michaelsen, 1910: 80.

TL. Vaxvella (Wakwella), Sri Lanka; TM. MNHU 7441; D. P. Western: Dt. Kalutara. Unspecified locality (Wickramaratne and Krishnarajah 2013); P. Southern: Dt. Galle: Vaxvella (Wakwella) (Michaelsen 1910); R. endemic.

50. Megascolex templetonianus Rosa, 1892

Megascolex templetonianus Rosa, 1892: 131.

TL. Ceylon (Sri Lanka); TM. ?; D. Ceylon (Sri Lanka) (Rosa 1892); P. Sabaragamuwa: Dt. Ratnapura: Pelmadulla (Gates 1945a), Varigama Kanda (Gates 1945a); P. Western: Dt. Colombo: Colombo (Ude 1893; Michaelsen 1897; Gates 1945a); R. endemic.

51. Megascolex varians insolitus Stephenson, 1915

Megascolex varians insolitus Stephenson, 1915: 86.

TL. Horton Plains, Sri Lanka; TM. ZSIC 6918; D. P. Central: Dt. Nuwara Eliya: Horton Plains (Stephenson 1915); R. endemic.

52. Megascolex varians simplex Michaelsen, 1897

Megascolex varians simplex Michaelsen, 1897: 207.

Megascolex annandalei Stephenson, 1913: 263.

Megascolex curtus Stephenson, 1913: 267.

TL. Nuwara Eliya, Sri Lanka. TM. Typus amissus; D. P. Central: Dt. Nuwara Eliya: Nuwara Eliya (Michaelsen 1897), Pattipola (Stephenson 1913); R. endemic.

\section{Megascolex varians varians Michaelsen, 1897}

Megascolex varians Michaelsen, 1897: 201.

Megascolex varians varians (Michaelsen): Blakemore 2007a: 37.

TL. Nuwara Eliya, Sri Lanka; TM. MNHU 7258 (holotypus) (siccum specimen), RNHL, MNHU 7466; D. P. Central: Dt. Kandy: Peradeniya (Michaelsen 1897);

Dt. Nuwara Eliya: Nuwara Eliya (Michaelsen 1897); R.endemic.

54. Megascolex (?) viridis (Schmarda, 1861)

Perichaeta viridis Schmarda, 1861: 13.

Megascolex (?) viridis (Schmarda): Michaelsen 1897: 242.

TL. Near Belligamme (Valikamam, Valligamam, Valikaamam), Sri Lanka; TM. MNHN; D. Southern Ceylon (Sri Lanka) (Schmarda, 1861); P. Northern: Dt. Jaffna: near Belligamme (Valikamam, Valligamam, Valikaamam) (Schmarda 
1861; Michaelsen 1897); P. Uva: Dt. Badulla: Badulla (Michaelsen 1900); R. endemic. Species inquirenda (Blakemore, 2007a). Michaelsen (1897) commented that, if it is not Megascolex, it should be assigned in Perionyx.

\section{Megascolex willeyi Michaelsen, 1909}

Megascolex willeyi Michaelsen, 1909: 96.

TL. Labugama, Sri Lanka; TM. Typus amissus; D. P. Western: Dt. Colombo: Labugama (Michaelsen 1909, 1910); R. endemic.

56. Megascolex zygochaetus Michaelsen, 1897

Megascolex zygochaetus Michaelsen, 1897: 199.

TL. Ratnapura, Sri Lanka; TM. WNHM 3977; D. P. Sabaragamuwa: Dt. Ratnapura: Ratnapura at the foot of Adam's peak (Michaelsen 1897); R. endemic.

57. Metaphire anomala (Michaelsen, 1907)

Pheretima anomala Michaelsen, 1907: 167.

Pheretima insolita Gates, 1925: 543

Pheretima anomalacentralis Stephenson, 1929: 234.

Metaphire anomala (Michaelsen): Sims and Easton 1972: 237.

TL. Botanic Gardens near Calcutta (Kolkata) (Blakemore 2012), India; TM. MNHU 4273, ZMUH 7185 (syntypus siccum specimen); D. P. ?Western: Unpsecified locality (Smaranayake 2013); R. exotic.

\section{Metaphire bahli (Gates, 1945)}

Pheretima bahli Gates, 1945a: 85

Metaphire bahli (Gates): Sims and Easton 1972: 239.

TL. Colombo $\left(6.92^{\circ} \mathrm{N}, 79.86^{\circ} \mathrm{E}\right)$, Sri Lanka; TM. Unknown (Nguyen et al. 2016);

D. P. Western: Dt. Colombo: Colombo (Gates 1945a), Dehiwala-Mount Lavinia (Szederjesi et al. 2019), Dt. Kalutara: Kalutara (Szederjesi et al. 2019), Wadduwa (Szederjesi et al. 2019); R. exotic.

59. Metaphire houlleti (Perrier, 1872)

Perichaeta houlleti Perrier, 1872: 99.

Perichaeta udekemi Michaelsen, 1892: 240

Pheretima crescentica Fedarb, 1898: 447.

Pheretima houlleti (Perrier): Michaelsen 1900: 273.

Metaphire houlleti (Perrier): Sims and Easton 1972: 238

For full list of synonyms see Blakemore (2012).

TL. Calcutta (Kolkata), India; TM. MNHN; D. P. Central: Dt. Kandy: Peradeniya (Michaelsen, 1903); P. Western: Dt. Colombo: Colombo museum garden (Michaelsen 1899), Dt. Gampaha: Heneratgoda (Henarathgoda) (Gates 1945a), Dt. Kalutara: Panadhure (Panadura) (Michaelsen 1910), unspecified locality 
(Wickramaratne and Krishnarajah 2013), Dt. Unspecified district and locality (Samaranayake 2013); R. exotic.

60. Nellogaster bahli (Stephenson, 1925)

Woodwardiella bahli Stephenson, 1925: 888.

Nellogaster bahli (Stephenson): Gates 1938: 428.

Notoscolex bahli (Stephenson): Blakemore 2007a: 43.

TL. Ceylon University College - Colombo, Sri Lanka; TM. BMNH 1933:2:23:37985, ZSIC 1207; D. P. Western: Dt. Colombo: Ceylon University College - Colombo (Stephenson 1925), Dt. Kalutara: Kalutara (Szederjesi et al. 2019); R. endemic. Blakemore (2007a) placed this species in Notoscolex. But recently Szederjesi et al. (2019) retained them in Nelloscolex by following Gates (1938), and stated that 'until a thorough revision of the Indian megascolecids is done we retain Gates (1938) combination'.

\section{Notoscolex ceylanensis (Michaelsen, 1897)}

Cryptodrilus ceylanensis Michaelsen, 1897: 183.

Notoscolex ceylanensis (Michaelsen): Michaelsen 1900: 194.

TL. Nuwara Eliya, Sri Lanka; TM. RNHL 1678, BMNH 1903:4:28:1; D. P. Central:

Dt. Nuwara Eliya: Hakgalla (Hakgala) (Gates 1941); Nuwara Eliya (Michaelsen 1897); R. endemic.

\section{Notoscolex crassicystis (Michaelsen, 1897)}

Cryptodrilus crassicystis Michaelsen, 1897: 194.

Notoscolex crassicystis (Michaelsen): Michaelsen 1900: 195.

TL. Nuwara Eliya, Sri Lanka; TM. Typus amissus; D. P. Central: Dt. Nuwara Eliya: Hakgalla (Hakgala) (Gates 1941), Nuwara Eliya (Michaelsen 1897); R. endemic.

\section{Notoscolex dambullaensis (Michaelsen, 1897)}

Cryptodrilus dambullaensis Michaelsen, 1897: 181.

Notoscolex dambullaensis (Michaelsen): Michaelsen 1900: 196.

TL. Dambulla, Sri Lanka; TM. Typus amissus; D. P. Eastern: Dt. Trincomalee: plains north of Trincomali (Trincomalee) (Michaelsen 1897); P. Central: Dt. Matale: plains north of Dambulla (Michaelsen 1897); R. endemic.

\section{Notoscolex decipiens (Michaelsen, 1897)}

Cryptodrilus decipiens Michaelsen, 1897: 197.

Notoscolex decipiens (Michaelsen): Michaelsen 1900: 191.

TL. Peradeniya?, Sri Lanka; TM. MZUT 145, MNHU 7405 (syntypus perditus); D. P. Eastern: Dt. Trincomalee: Kaniya (Kanniya) (Michaelsen 1910); P. Central: Dt. Kandy: Peradeniya (Michaelsen 1897, 1910); P. Uva: Dt. Badulla: East of Badulla (Michaelsen 1900), Avissavela; P. Western: Dt. Colombo: Avissavela (Avissavella) (Michaelsen 1910), Colombo Museum garden (Michaelsen 1899), 
Dt. Gampaha: Heneratgoda (Henarathgoda) (Gates 1941), Dt. Kalutara: Panadhure (Panadura) (Michaelsen 1910); R. endemic.

\section{Notoscolex gravelyi Stephenson, 1916}

Notoscolex gravelyi Stephenson, 1916: 325.

TL. Lady Blake’s Drive - Kandy, Sri Lanka; TM. ZSIC 6569; D. P. Central: Dt. Kandy: Lady Blake's Drive - Kandy (Stephenson 1916); R. endemic.

66. Notoscolex hakgallanus Gates, 1941

Notoscolex hakgallanus Gates, 1941: 43.

TL. Hakgalla (Hakgala), Sri Lanka; TM. ?; D. P. Central: Dt. Nuwara Eliya: Hakgalla (Hakgala (Gates 1941); R. endemic.

\section{Notoscolex jacksoni (Beddard, 1890)}

Deodrilus jacksoni Beddard, 1890: 467.

Cryptodrilus jacksoni (Beddard): Michaelsen 1897: 190.

Notoscolex jacksoni (Beddard): Michaelsen 1900: 196.

TL. Ceylon (Sri Lanka); TM. Typus amissus; D. Ceylon (Sri Lanka) (Beddard 1890); P. Eastern: Dt. Trincomalee: Trincomali (Trincomalee) (Michaelsen 1897); P. Central: Dt. Nuwara Eliya: Nuwara Eliya (Michaelsen 1897); R. endemic.

68. Notoscolex kraepelini (Michaelsen, 1904)

Trinephrus kraepelini Michaelsen, 1904: 128.

Notoscolex kraepelini (Michaelsen): Stephenson 1923: 211.

TL. Between Matale and Anuradhapura, Sri Lanka; TM. ZMUH 6475; D. P. Central: ?Dt.: Between Matale and Anuradhapura (Michaelsen 1904); R. endemic.

69. Notoscolex lautus Gates, 1945

Notoscolexlautus Gates, 1945a: 71.

TL. Varigama Kanda, Sri Lanka; TM. ?; D. P. Sabaragamuwa: Dt. Ratnapura: Varigama Kanda (Gates 1945a); R. endemic.

70. Notoscolex plenus Gates, 1945

Notoscolexplenus Gates, 1945a: 73.

TL. Varigama Kanda, Sri Lanka; TM. ?; D. P. Sabaragamuwa: Dt. Ratnapura: Varigama Kanda (Gates 1945a); R. endemic.

71. Notoscolex sarasinorum (Michaelsen, 1897)

Cryptodrilus sarasinorum Michaelsen, 1897: 177.

Notoscolex sarasinorum (Michaelsen): Michaelsen 1900: 192.

Woodwardia sarasinorum (Michaelsen): Stephenson 1923: 188 
Woodwardiella sarasinorum (Michaelsen): Blakemore 2007a: 50.

TL. ? Peradeniya, Sri Lanka; TM. Typus amissus; D. P. Central: Dt. Kandy: Probably from Peradeniya (Michaelsen 1897); R. endemic. Blakemore (2007a) placed this species in Woodwardiella. But following Gates (1960), we retain it in Notoscolex.

\section{Notoscolex termiticola Michaelsen, 1910}

Notoscolex termiticola Michaelsen, 1910: 63.

TL. Peradeniya, Sri Lanka; TM. Typus amissus; D. P. Central: Dt. Kandy: Peradeniya (Michaelsen 1910); R. endemic.

\section{Notoscolex trincomaliensis (Michaelsen, 1897)}

Cryptodrilus trincomaliensis Michaelsen, 1897: 188.

Notoscolex trincomaliensis (Michaelsen): Michaelsen 1900: 190.

TL. Trincomali (Trincomalee), Sri Lanka; TM. Typus amissus; D. P. Eastern: Dt.

Trincomalee: plains north of Trincomali (Trincomalee) (Michaelsen 1897); P. Central: Dt. Matale: plains north of Dambulla (Michaelsen 1897); R. endemic.

\section{Notoscolex uzeli (Michaelsen, 1903)}

Plutellus uzeli Michaelsen, 1903: 4.

Woodwardia uzeli (Michaelsen): Michaelsen 1910: 57.

Notoscolex uzeli (Michaelsen): Gates 1960: 240.

Woodwardiella uzeli (Michaelsen): Blakemore 2007a: 50.

TL. Peradeniya, Sri Lanka; TM. Typus amissus; D. P. Central: Dt. Kandy: Peradeniya (Michaelsen 1903, 1910); P. Uva: Dt. Badulla: Avissavela (Michaelsen 1910); R. endemic. Blakemore (2007a) placed this species in Woodwardiella, but following Gates (1960), we retain it in Notoscolex.

\section{Perionyx ceylanensis Michaelsen, 1903}

Perionyx ceylanensis Michaelsen, 1903: 6.

TL. Peradeniya, Sri Lanka; TM. Typus amissus; D. P. Central: Dt. Kandy: Peradeniya (Michaelsen 1903); P. ?Western: Unspecified locality (Samaranayake 2013); P. Southern: Dt. Galle: Point de Galle (Michaelsen 1910); E. India (Kathireswari and Julka 2008); R. near endemic.

\section{Perionyx excavatus Perrier, 1872}

Perionyx excavatus Perrier, 1872: 126.

Perionyx gruenewaldi Michaelsen, 1891: 33.

Perionyx parvulus Stephenson, 1916: 321.

Full list of synonyms see Blakemore (2012).

TL. Saigon, Vietnam; TM. MNHN; D. P. Central: Dt. Kandy: Kandy (Michaelsen 1909), Peradeniya (Michaelsen 1903); P. ?Western: Unspecified locality (Samaranayake 2013); R. exotic. 
77. Perionyx polythecus Stephenson, 1923

Perionyx polytheca Stephenson, 1923: 351.

Perionyx polythecus (Stephenson): Blakemore 2007a: 46.

TL. Peradeniya, Sri Lanka; TM. ?; D. P. Central: Dt. Kandy: Peradeniya (Michaelsen 1903); R. endemic.

78. Polypheretima elongata (Perrier, 1872)

Perichaeta elongata Perrier, 1872: 124.

Perichaeta acystis Beddard 1895:423

Amynthas elongata (Perrier): Beddard 1900: 650.

Pheretima elongata (Perrier): Michaelsen 1900: 265.

Metapheretima elongata (Perrier): Sims and Easton 1972: 205.

Polypheretima elongata (Perrier): Easton 1979: 53.

For full list of synonyms see Blakemore (2012).

TL. Peru; TM. MNHN 633-44, but according to Nguyen et al. (2016) MNHN (AE 633-646); D. P. Central: Dt. Kandy: Kandy (Michaelsen 1909); P. Western: Dt. Kalutara: Panadhure (Panadura) (Michaelsen 1910), unspecified locality (Samaranayake 2013); R. exotic.

\section{Polypheretima taprobanae (Beddard, 1892)}

Perichaeta taprobanae Beddard, 1892: 163.

Perichaeta pauli Michaelsen, 1897: 234.

Amynthas taprobanae (Beddard): Beddard 1900: 648.

Pheretima taprobanae (Beddard): Michaelsen 1903: 12.

Metapheretima taprobanae (Beddard): Sims and Easton 1972: 233.

Polypheretima taprobanae (Beddard): Easton 1979: 45.

For further synonyms see Easton (1979) and Blakemore (2012).

TL. ? Peradeniya, Sri Lanka; TM. BMNH 1904: 10:5:163-4, 1972:1:6-11; D. Ceylon (Sri Lanka) (Beddard 1892); P. Central: Dt. Kandy: near Peradeniya (Michaelsen 1897, 1903; Gates 1945a); P. Western: unspecified locality (Samaranayake 2013), Dt. Kaluatara: Matugama (Szederjesi et al. 2019); R. exotic.

80. Pontodrilus agnesae Stephenson, 1915

Pontodrilus agensae Stephenson, 1915: 61.

TL. Horton Plains, Sri Lanka; TM. ZSIC 6183, 6190, BMNH 1933:5:25:63; D. P. Central: Dt. Nuwara Eliya: Elk Plains (Stephenson 1915), Horton Plains (Stephenson 1915); R. endemic.

\section{Pontodrilus litoralis (Grube, 1855)}

Lumbricus litoralis Grube, 1855: 127.

Pontodrilus bermudensis Beddard, 1891: 96. 
Pontodrilus laccadivensis Beddard, 1903: 374.

Further synonymies in Gates (1972), Blakemore (2012).

TL. Villa Franca of French Riviera, France; TM. MNHU 216 (syntypus siccum specimen), WUMW 506 (paratypus); D. P. Northern: Dt. Jaffna: Belligamme (Valikamam, Valligamam, Valikaamam) (Michaelsen 1897), Pungudutivu (Atputhanathan 1968), Thondaimanar (Thondamannar, Thondamanaru) (Atputhanathan 1968); P. Western: Dt. Gampaha: Negombo (Atputhanathan 1968); R.exotic.

\section{Discussion}

Until recently it is thought that Sri Lanka had 63 species of earthworms, of which 48 are endemic (Szederjesi et al. 2019). The present checklist reveals the occurrence of 81 species and subspecies of earthworms belonging to 21 genera and 8 families from Sri Lanka. It is rather a high species diversity in view of Sri Lanka's small geographical area. It is noteworthy that earthworm fauna of Sri Lanka possesses a close relation to the Western Ghats Mountains of India, especially of the Kerala state (Narayanan et al. 2016).

About $83 \%$ (67 species, including both native and exotic) of the species belong to Megascolecidae family and rests of the families are represented by very few species. Family Benhamiidae is represented by 4 species, Lumbricidae represented with 3 species and Moniligastridae and Ocnerodrilidae are represented by 2 each, whereas Almidae, Rhinodrilidae and Eudrilidaeare represented by single species each. Among these, Benhamiidae, Eudrilidae, Lumbricidae, Ocenrodrilidae and Rhinodrilidae are exotic to Sri Lanka. Genera with higher number of species and subspecies are Megascolex (37 species) followed by Notoscolex (14), together they form nearly 63\% of all the species recorded from the country.

Province wise distribution of species shows that Central province tops the list with 58 species, followed by Western with 28, Sabaragamuwa with 11 and as of now no species has been reported from the North Western province (Fig. 3). Difference in number of species is definitely due to uneven exploration intensity. From the figure 3, it is clear that except Central province, much of the other provinces are not surveyed systematically. Several areas are yet to be explored in the country, especially the biodiversity-rich national parks, wildlife sanctuaries, which along with other forest tracts of the Sri Lanka are considered refugia of the relic biota of the former Indian plate.

As in the case of many other taxa, earthworms of Sri Lanka and Western Ghats of India show an exceptionally high level of endemism ( $71.6 \%$ and about $77 \%$ (Narayanan et al. 2020, 2021) respectively), which may be due to the fact that these two areas of the Indian plate, which were the part of the ancient super continent Gondwanaland, had never been fully submerged under the sea in the various geological time periods (Illangasinghe et al. 1999; Julka and Paliwal 2005). Of the estimated 9 genera with 


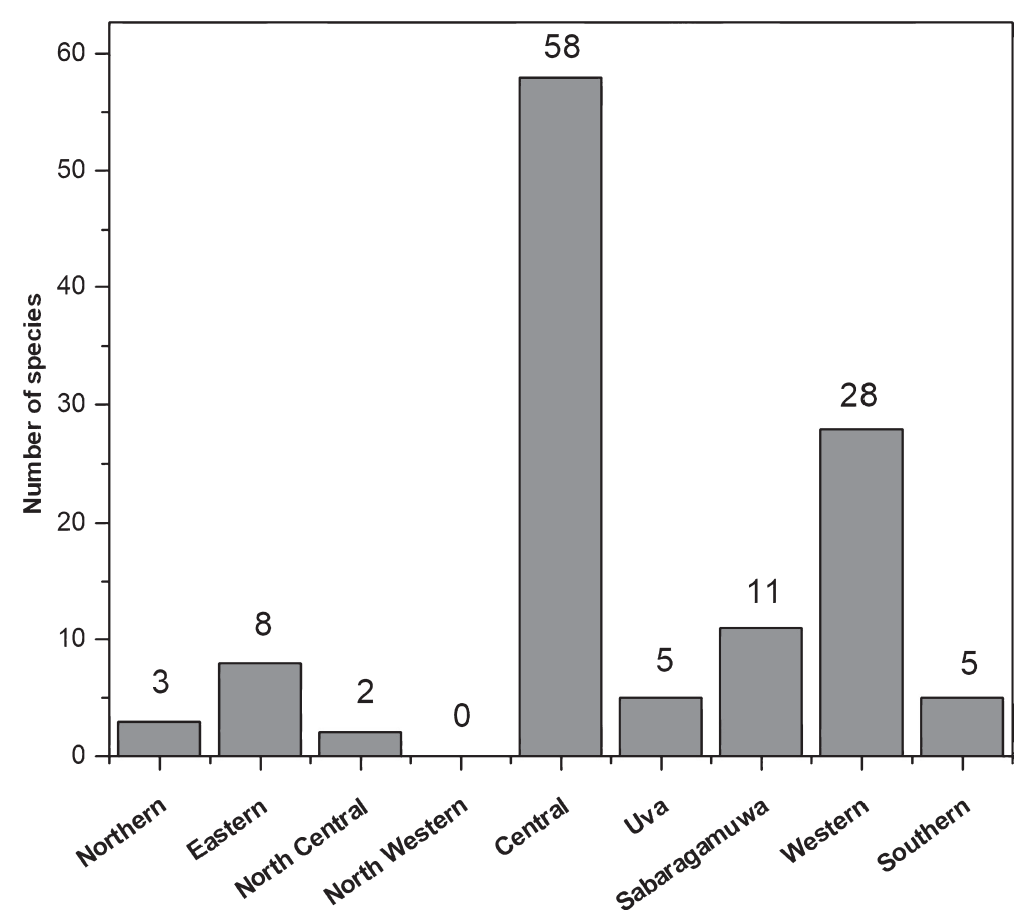

Figure 3. Valid earthworm species recorded from each provinces of Sri Lanka.

endemic taxa in Sri Lanka, Megascolex is dominant with 36 species and subspecies followed by Notoscolex (14 species), Drawida (2), Argilophilus (2), Glyphidrilus, Pontodrilus and Perionyx with one species each. Taxonomic history of Plutellus and Argilophilus is complicated (see Tiwari et al. 2020). Gates (1972), recommended placing Oriental species of Plutellus in Argilophilus. However, the presence of the North American Argilophilus in the Indian subcontinent is dubious. Later Blakemore (2006) commented that the Indo-Oriental Argilophilus species should be placed in another genus, probably a new one. Further molecular work should be carried out to clarify the status of these species and thus require a taxonomic revision of species of Plutellus and Argilophilus. Megascolex insignis and Perionyx ceylanensis are the near endemics, since these are also known to occur in India. A number of genera (Megascolex and Notoscolex) have very ancient lineages that might have their origin in Gondwanaland. Given the high rate of endemism, earthworm fauna of Western Ghats-Sri Lanka biodiversity hotspot is of immense bio-geographical significance.

About 26\% (21 species) of the earthworm fauna of Sri Lanka is of exotic origin, which were presumably introduced from the neighbouring bio-geographical regions or from India by human commerce. These are: Dichogaster affinis, D. annae, D. bolaui, D. saliens, Eudrilus eugeniae, Nematogenia panamaensis (Ethiopian region); Ocnerodrilus occidentalis (Meso America or Neotropical region); Pontoscolex corethrurus (Neotropical region); Aporrectodea caliginosa, Eisenia fetida, Octolasion 
cyaneum (West Palaearctic region); Amynthas corticis, A. gracilis, Metaphire anomala, M. bahli, M. houlleti, Polypheretima elongata, P. taprobanae (mainly from Southeast Asian region); Pontodrilus litoralis (possibly southern India or Australia, or both (Blakemore 2007b); Lampito mauritii, Perionyx excavatus (India). According to Blakemore (2012) Amynthas genus is mainly found in the Oriental and Oceania regions, the natural distribution is unclear due to disproportionately high numbers of peregrine species; hence here we treated it as an exotic species.

Several species are known only from the original description, and most of them were recorded more than a century ago. Since then studies on the earthworm fauna of the country have been limited and no further studies have been carried out after the independence of this island country. Hence, due to the lack of revisionary works, the taxonomic status of these species, their level of morphological variation etc. cannot be considered as confirmed. The Western Ghats and Sri Lanka have similar levels of endemism among various taxa such as trees, bryophytes, land snails, odonates, fishes, amphibians and reptiles, though the fauna of the wet evergreen forest zones have been found to be quite distinctive and forms endemic clades, where as the fauna and flora of lowland dry forests seems more similar (Bossuyt et al. 2004; Gunawardene et al. 2007; Sudasinghe et al. 2020). Earthworms are also not an exception for this; Megascolex and Notoscolex species show high level of endemism in Sri Lanka and Western Ghats south of Palghat (Palakkad) gap (Narayanan et al. 2016). Recent studies on various taxa indicate that Sri Lankan fauna is derived from evolutionary diverse ancestral stock from the Indian mainland mostly before the Pleistocene epoch and underwent in-situ radiation within the island (Bossuyt et al. 2004; Biswas 2008). Hence further comparative studies are to be conducted between the earthworms of the Western Ghats of India and Sri Lankan highlands in order to understand the evolutionary history of the earthworms.

Sri Lanka has been densely settled since early times, even though much of the areas were almost covered by tropical forests, however in the last 150 years extensive deforestation has taken place as a result of European colonisation, urbanisation, logging, tea and rubber plantation expansion (Samaranayake and Moyle 1982; Lindström 2011). According to McNeely et al. (1990) the rate of forest and wildlife habitat loss in Sri Lanka is considered as one among the highest in southern Asia. Hence, many species described early in the last century might have gone extinct. Systematic collection would help to know the present-day existence of these species in Sri Lanka. Further, faunistic surveys of under explored and unexplored areas in the country would bring to light additional species.

\section{Acknowledgements}

We are expressing our sincere gratitude to Dr Karunakaran Akhildev for preparing the map of Sri Lanka. 


\section{References}

Atputhanathan M (1968) Study on two annelids worms Pontodrilus bermudensis, Beddard, 1891, Odontosyllis gravelyi, Fauvel, 1928, Hydrobiological Survey of the Thondaimannar Lagoon Bulletin no. 6. Hydrobiological Survey Research Council of the Northern Province Science Teachers Association, 73 pp.

Backlund HO (1948) Eisenia fasciata n. sp. a new lumbricid from Sweden. Kungliga Fysiografi ska Sallskapets Handlingar 18(6): 1-5.

Beddard FE (1882) On the anatomy and histology of Pleurochaeta moseleyi. Transactions of the Royal Society of Edinburgh 30(2): 481-509. doi:10.1017/S0080456800026557

Beddard FE (1886) Notes on earthworms from Ceylon and the Philippine Islands, including a description of two new species. The Annals and Magazine of Natural History, Series 5 (98): 89-98. http://dx.doi.org/10.1080/00222938609460120

Beddard FE (1890) On the occurence of new genus of Oligochaeta (Deodrilus), and on the presence of anal nephridia in Acanthodrilus. Quarterly Journal of Microscopical Science 121: 467-488.

Beddard FE (1892) On some species of the genus Perichaeta (sensu stricto). Proceedings of the Zoological Society of London 1892: 153-172.

Beddard FE (1893) On some new species of earthworms from various parts of the world. Proceedings of the Zoological Society of London 1892: 666-706.

Beddard FE (1895) A monograph of the Order Oligochaeta. Oxford, Clarendon Press, 769 pp. Beddard FE (1900) A revision of the earthworms of the genus Amyntas (Perichaeta). Proceedings of the Zoological Society of London 69(4): 609-652.

Beddard FE (1903) The earthworms of the Maldive and Laccadive Islands. In: Gardiner JS (Ed.) The fauna and geography of the Maldive and Laccadive Archipelagoes, $374 \mathrm{pp}$.

Biswas S (2008) Did biotic impoverishment facilitate phenomenal diversification in Sri Lanka? Current Science 95(8): 1021-1025.

Blakemore RJ (2000) Native earthworms (Oligochaeta) from Southeastern Australia, with the description of fifteen new species. Records of the Australian Museum 52: 187-222.

Blakemore RJ (2005) Whither Octochaetidae? - its family status reviewed. In: Pop AA, Pop VV (Eds) Advances in Earthworm Taxonomy II. Proceedings IOTM2, Cluj University Press. Romania, 63-84.

Blakemore RJ (2006) Checklist of Myanmar taxa updated from Gates' (1972) "Burmese Earthworms". Online at http://www.annelida.net/earthworm/Myanmar\%20taxa\% 20updated\%20from\%20Gates.pdf (accessed: 12 January 2020).

Blakemore RJ (2007a) Checklist of Indian earthworms. In: A series of Searchable Texts on Earthworm Biodiversity, Ecology and Systematics from Various Regions of the World $-3^{\text {rd }}$ Edition. Online at: http://www.annelida.net/earthworm/Indian.pdf. (accessed: 2 February 2016).

Blakemore, RJ (2007b) Origin and means of dispersal of cosmopolitan Pontodrilus litoralis (Oligochaeta: Megascolecidae). European Journal of Soil Biology 43: S3-S8. doi:10.1016/j. ejsobi.2007.08.041 
Blakemore RJ (2012) Cosmopolitan Earthworms - an Eco-Taxonomic Guide to the Peregrine Species of the World ( $5^{\text {th }}$ edn.). VermEcology So(i)lutions, Yokohama, Japan, $850 \mathrm{pp}+$ 350 Figs and Internet links.

Blakemore RJ (2013) The major Megadrile families of the world reviewed again on their taxonomic types (Annelida: Oligochaeta: Megadrilacea). Opuscula Zoologica Budapest 44(2): 107-127.

Bossuyt F, Meegaskumbura M, Beenaerts N, Gower DJ, Pethiyagoda R, Roelants K, Mannaert A, Wilkinson M, Bahir MM, Manamendra-Arachchi K, Ng PKL, Schneider J, Oommen OV, Milinkovitch MC (2004). Local endemism within the Western Ghats-Sri Lanka biodiversity hotspot. Science 306(5695): 479-481. https://doi.org/10.1126/science.1100167 Bouché MB (1972) Lombriciens de France. Écologie et Systématique. INRA Publication. Institut National des Recherches Agriculturelles, Paris. 1-671 pp. [in French]

Bourne AG (1891) On Megascolex caeruleus, Templeton, from Ceylon; together with a theory of the course of the blood in earthworms. Quarterly Journal of Microscopical Science 32: $49-87$.

Burmeister H (1835) Zoologischer Hand-Atlas zum Schulgebrauch und Selbstunterricht. Georg Reiner, Berlin, 42 p. [in German]

Csuzdi C (1995) A catalogue of Benhamiinae species (Annelida: Oligochaeta: Acanthodrilidae). Annalen des Naturhistorischen Museums in Wienna 97B: 99-123.

Csuzdi C (1996) Revision der Unterfamilie Benhamiinae Michaelsen, 1897 (Oligochaeta: Acanthodrilidae). Mitteilungen aus dem Zoologischen Museum Berlin 72: 347-367. [in German]

Easton EG (1979) A revision of the 'acaecate' earthworms of the Pheretima group (Megascolecidae: Oligochaeta): Archipheretima, Metapheretima, Planapheretima, Pleionogaster and Polypheretima. Bulletin of the British Museum (Natural History) Zoology 35: 1-126.

Easton EG (1982) Australian pheretimoid earthworms (Megascolecidae: Oligochaeta): a synopsis with the description of a new genus and five new species. Australian Journal of Zoology 30: 711-735.

Easton EG (1983) A guide to the valid names of Lumbricidae (Oligochaeta). In: Satchell JE (Ed.) Earthworm Ecology - From Darwin to Vermiculture. Chapman \& Hall, London, 475-487 pp.

Eisen G (1878) On the anatomy of Ocnerodrilus. Nova Acta Regiae Societatis Scientiarum Upsaliensis 10(3): 1-12.

Eisen G (1900) Researches on American Oligochaeta, with special reference to those of the Pacific Coast and adjacent islands. Proceedings of the California Academy of Sciences 2(3): 85-276.

Epa R, Perea N, Manamendra-Arachchi K, Meegaskumbara M (2012) Sri Lanka’s Aruwakkalu fossil deposit dates to the Burdiggalian age. Ceylon Journal of Science (Biological Sciences) 40: 163-174. https://doi.org/10.4038/cjsbs.v40i2.3933.

Fedarb SM (1898) On some earthworms from British India. Proceedings of the Zoological Society of London 1898: 445-450. 
Fender WM, McKey-Fender D (1990) Oligochaeta: Megascolecidae and other earthworms from western North America. In: Dindal DL (Ed.) Soil Biology Guide, John Wiley and Sons, New York, pp. 357-378.

Gates GE (1938) Indian earthworms. IV. The genus Lampito Kinberg. V. Nellogaster, gen. nov., with a note on Indian species of Woodwardiella. Records of the Indian Museum 40(4): 403-429.

Gates GE (1941) On some earthworms from Ceylon. Spolia Zeylanica 23: 27-60.

Gates GE (1945a) On some earthworms from Ceylon. II. Spolia Zeylanica 24: 70-90.

Gates GE (1945b) On some Indian earthworms. Proceedings of the Indian Academy of Science, Section B, 30(5): 208-283.

Gates GE (1959) On a taxonomic puzzle and the classification of the earthworms. Bulletin of the Museum of Comparative Zoology, Harvard 121(6): 229-261.

Gates GE (1960) On Burmese earthworms of the family Megascolecidae. Bulletin of the Museum of Comparative Zoology, Harvard 123(6): 203-282.

Gates GE (1972) Burmese earthworms, an introduction to the systematic and biology of the megadrile oligochaetes with special reference to southeast Asia. Transactions of the American Philosophical Society 62(7): 1-326.

Grube E (1855) Beschreibungen neuer oder wenig bekannter Anneliden. Archiv fur Naturgeschichte 21: 81-136. [in German]

Gunatilleke N, Pethiyagoda R, Gunatilleke S (2008) Biodiversity of Sri Lanka. Journal of the National Science Foundation of Sri Lanka 36: 25-62.

Gunawardene NR, Daniels AED, Gunatilleke IAUN, Gunatilleke CVS, Karunakaran PV, Nayak KG, Prasad S, Puyravaud P, Ramesh BR, Subramanian KA, Vasanthy G (2007) A brief overview of the Western Ghats - Sri Lanka biodiversity hotpsot. Current Science 93(11): 1567-1572.

Illangasinghe W, Fujiwara K, Saito H (1999) A preliminary study of forests in Sri Lanka. Bulletin, Institute of Environmental Science and Technology, Yokohama National University 25: 9-38.

James SW, Bartz MLC, Satnton DWG, Conrado AC, Dupont L, Taheri S, Da Silva E, Cunha L, Brown GG (2019) A neotype for Pontoscolex corethrurus (Müller, 1857) (Clitellata). Zootaxa 4545(1): 124-132. https://doi.org/10.11646/zootaxa.4545.1.7

James SW, Gamiette F (2016) New species of Dichogaster Beddard, 1888 (Clitellata: Benhamiidae) with additional records of earthworms from Guadeloupe (French West Indies). Zootaxa 4178 (3): 391-408. https://doi.org/10.11646/zootaxa.4178.3.5

Julka JM (1988) The Fauna of India and Adjacent Countries. Megadrile Oligochaeta (Earthworms). Haplotaxida: Lumbricina: Megascolecoidea: Octochaetidae. Zoological Survey of India, Calcutta, 400 pp.

Julka JM, Paliwal R (2005) Distribution of earthworms in different agro-climatic regions of India. In: Ramakrishnan PS, Saxena KG, Swift MJ, Rao KS, Maikhuri RK (Eds) Soil Biodiversity, Ecological Processes and Landscape. Oxford and ABH Publications Co Pvt. Ltd, New Delhi, 3-13 pp.

Kathireswari P, Julka JM (2008) First record of earthworms Perionyx ceylanensis and Eukerria kukenthali (Annelida: Oligochaeta) from mainland India. Megadrilogica 12(8): 117-120. 
Kinberg JGH (1867) Annulata nova. Ofversigt af Kongl. Vetenskaps-Akademiens Förhandlingar, Stockholm 23: 97-103. [in Swedish]

Lindström S (2011) Tropical deforestation in Sri Lanka, a minor field study investigating the impact of small-scale farmers. Bachelor thesis, University of Gothenburg.

McNeely JA, Miller KR, Reid WV, Mittermeier RA, Werner TB (1990) Conserving the world's biological diversity. World Conservation Union, Gland, Switzerland.

Michaelsen W (1890) Beschreibung der von Herrn Dr. Stuhlmann im Mudungsgebiet des Sambesi gesammelten Terricolen. Jahrbuch der hamburgischen wissenschaftlichen Anstalten 7: 1-30. [in German]

Michaelsen W (1891) Oligochaeten des Naturhistorischen Museums in Hamburg. IV. Jahrbuch der hamburgischen wissenschaftlichen Anstalten 8: 3-42. [in German]

Michaelsen W (1897) Die terricololenfauna Ceylons. Mitteilungen aus dem Naturhistorischen Museum in Hamburg 2: 157-250. [in German]

Michaelsen W (1899) Beitrage zur Kenntniss der Oligochäten. Zoologische Jahrbücher, Abteilung für Systematik 12: 105-144. [in German]

Michaelsen W (1900) Das Tierreich 10: Vermes, Oligochaeta. Friedländer and Sohn, Berlin, 575pp. [in German]

Michaelsen W (1903) Oligochaeten von Peradeniya auf Ceylon, ein beitrag sur kenntnis des einflusses botanischer garten auf die einschleppung peregriner thiere. Sitzungsberichte der Koniglischen Bohmischen Gesellschaft der Wissenschaften. Mathematischnaturwissenschaftliche Classe, 1-16 pp. [in German]

Michaelsen W (1904) Über eine Trinephrus - Art von Ceylon. Mitteilungen aus dem Naturhistorischen Museum in Hamburg 21: 157-250. [in German]

Michaelsen W (1907) Neue Oligochäten von Vorder-Indien, Ceylon, Birma und den Andaman-Inseln. Jahrbuch der hamburgischen wissenschaftlichen Anstalten 24: 143-188. [in German]

Michaelsen W (1909) The Oligochaeta of India, Nepal, Ceylon, Burma and the Andaman Islands. The Memoirs of the Indian Museum 1: 103-253.

Michaelsen W (1910) Die Oligochäten fauna der vorderindisch-ceylonischen region. Abdruck Verhandelingen der Naturwissenschaftlichen Vereins zu Hamburg 19(5): 1-108. [in German]

Michaelsen W (1922) Oligochätenvon westlichen Vorderindien und ihre Beziehungen zur Oligochätenfauna von Madagaskar und den Seychellen. Jahrbuch der hamburgischen wissenschaftlichen Anstalten 38: 27-68. [in German]

Mittermeier RA, Turner WR, Larsen, FW, Broos TM, Gscon C (2011) Global biodiversity conservation: the critical role of hotspots. In: ZaChos FE, Habel JC (Eds) Biodiversity Hotspots, Springer Publishers, London, 3-22 pp.

Müller F (1857) Lumbricus corethrurus, Busten-schwanz. Archiv für Naturgeschichte 23(1): 113-116. [in German]

Myers N, Mittermeier RA, Mittermeier CG, da Fonseca GAB, Kent J (2000) Biodiversity hotspots for conservation priorities. Nature 403: 853-858. 
Narayanan SP, Sathrumithra S, Christopher G, Thomas AP, Julka JM (2016) Checklist of the earthworms (Oligochaeta) of Kerala, a constituent of Western Ghats biodiversity hotspot, India. Zootaxa 4193(1): 117-137. http://doi.org/10.11646/zootaxa.4193.1.5

Narayanan SP, Paliwal R, Kumari S, Ahmed S, Thomas AP, Julka JM (2020) Annelida: Oligochaeta. In: Director (Ed) Faunal Diversity of Biogeographic Zones of India: Western Ghats. Zoological Survey of India, Kolkata, 87-102 pp.

Narayanan SP, Sathrumithra S, Anuja R, Christopher G, Thomas AP, Julka JM (2021) Three new species and four new species records of earthworms of the genus Moniligaster Perrier, 1872 (Clitellata: Moniligastridae) from Kerala region of the Western Ghats Biodiversity Hotspot, India. Zootaxa 4949(2): 381-397. https://doi.org/10.11646/zootaxa.4949.2.11

Nguyen TT, Nguyen AD, Tran BTT, Blakemore RJ (2016) A comprehensive checklist of earthworm species and subspecies from Vietnam (Annelida: Clitellata: Oligochaeta: Almidae, Eudrilidae, Glossoscolecidae, Lumbricidae, Megascolecidae, Moniligastridae, Ocnerodrilidae, Octochaetidae). Zootaxa 4110(1): 1-92. http://doi.org/10.11646/ zootaxa.4140.1

Pavlíček T, Hadid Y, Csuzdi C (2012) Opening Pandora's box: clitellum in phylogeny and taxonomy of earthworms. Zoology in the Middle East, Supplementum, 4: 31-46.

Perera GAD (2001) The secondary forest situation in Sri Lanka: a review. Journal of Tropical Forest Science 13(4): 768-785.

Perrier E (1871) Description of Eudrilus and Eu. decipiens. Les Comptes Rendus de l'Académie des sciences, Paris 73: 1175-1176. [in French]

Perrier E (1872) Recherche pour servir à l'historie des Lombriciens terrestres. Nouvelles Archives du Museum d'Histoire Naturelle Paris, 8: 5-198. [in French]

Perrier E (1875) Sur les vers de terre des iles Philippines et de Cochinchine. Compte rendu hebdomadaire des seances de I'Academie des Sciences Paris (D) 81: 1043-1046. [in French]

Reynolds JW, Wetzel MJ (2017) Nomenclatura Oligochaetologica - a catalogue of names, descriptions and type specimens. Editio Secunda. Online at http://wwx.inhs.illinois.edu/ people/mjwetzel/nomenoligo. (accessed: 6 September 2017).

Rosa D (1891) Die exotischen Terricolen des k. k. naturhistorischen Hofmuseums. Annalen des (K. K.) Naturhistorischen (Hof Museums Wein) 6: 379-406. [in German]

Rosa D (1892) Megascolex templetonianus n. sp. (Diagnosi preventiva). Bollettino dei Musei di Zoologia ed Anatomia Comparata della R. Universitá di Torino 7(131): 1-2. [in Italian]

Rosa D (1893) Revisione dei Lumbricidi. Memorie della Reale Accademia delle Scienze di Torino Serie 2, 43: 399-476. [in Italian]

Rosa D (1894) Perichetini nuovi o meno noti. Atti della R. Accademia delle Scienze di Torino 29: 1-18. [in Italian]

Samaranayake FR, Moyle PB (1982) Conservation of freshwater fishes of Sri Lanka. Biological Conservation 22: 181-195.

Samaranayake JWK (2013). Present status of Annelida: Oligochaeta (earthworms) diversity in Sri Lanka. In: Anonymous (Ed.) Conservation and Sustainable Use of Soil Biodiversity in Sri Lanka, Proceedings of the National Symposium on soil biodiversity - 2013. Biodiversity Secretariat, Ministry of Environment \& Renewable Energy, Sri Lanka, 37-40 pp. 
Savigny JC (1826) Analyses des travaux de l' Academie Royale des Sciences pendant l'année 1821, partie physique. In: Cuvier M, le Baron G (Eds) Mémoires de l'Académie des Sciences, France, 5: 176-184. [in French]

Schmarda LK (1861) Neue wirbellose thiere beobachtet und gesammelt auf einer Reise um die Erder 1853 bis 1857. Erster band. Turbellarian, Rotatorien und Anneliden. Leipzig, Wilhelmann, 161 pp. [in German]

Sims RW, Easton EG (1972) A numerical revision of the earthworm genus Pheretima auct.

(Megascolecidae: Oligochaeta) with the recognition of new genera and an appendix on the earthworms collected by the Royal Society North Borneo Expedition. Biological Journal of the Linnaean Society 4: 169-268.

Stephenson J (1913) On a collection of Oligochaeta from, mainly from Ceylon. Spolia Zeylanica 3: 251-276.

Stephenson J (1915) On some Indian Oligochaeta, mainly from Southern India and Ceylon. Memoirs of the Indian Museum 6: 35-108.

Stephenson J (1916) On a collection of Oligochaeta belonging to Indian Museum. Records of the Indian Museum 12: 299-354.

Stephenson J (1923) The Fauna of British India, including Ceylon and Burma - Oligochaeta. Taylor and Francis, London, 518 pp.

Stephenson J (1925) Oligochaeta from various regions, including those collected by the Mount Everest expedition 1924. Proceedings of the Zoological Society of London 95(3): 879-907. Stephenson J (1926) Descriptions of the Oligochaeta I. Records of the Indian Museum 28: 249-267.

Sudasinghe H, Pethiyagoda R, Meegaskumbura M (2020) A molecular phylogeny of the genus Laubuka (Teleostei: Cyprinidae) in Sri Lanka reveals multiple origins and a cryptic species. Systematics and Biodiversity https://doi.org/10.1080/14772000.2020.1771468

Szederjesi T, Pavlíček T, Csuzdi C (2019) New earthworm records from several Indian Ocean islands (Clitellata, Megadrili). Opuscula Zoologica Budapest 50(2): 145-155. http://dx.doi. org/10.18348/opzool.2019.2.145

Templeton R (1844) Description of Megascolex caeruleus. Proceedings of the Zoological Society of London 12: 89-91.

Tiwari N, Paliwal R, Rashid A, Yadav S (2020) Checklist of earthworm species (Oligochaeta) of the North Eastern Region of India. Zootaxa 4772(2): 277-305. https://doi.org/10.11646/ zootaxa.4772.2.3

Ude H (1893) Beiträge zur Kenntnis ausländischer Regenwürmer. Zeitschrift für Wissenschaftliche Zoologie 57: 57-75. [in German]

Wikramaratne MWD, Krishnarajah SR (2013) Present status of Annelida: Oligochaeta (earthworms) diversity in Sri Lanka. In: Anonymous (Ed.) Conservation and Sustainable Use of Soil Biodiversity in Sri Lanka, Proceedings of the National Symposium on soil biodiversity - 2013. Biodiversity Secretariat, Ministry of Environment \& Renewable Energy, Sri Lanka, 56-60 pp. 